\title{
Some results on packing in Orlicz sequence spaces
}

\author{
by \\ Y. Q. YAN (Suzhou)
}

\begin{abstract}
We present monotonicity theorems for index functions of $N$-fuctions, and obtain formulas for exact values of packing constants. In particular, we show that the Orlicz sequence space $l^{(N)}$ generated by the $N$-function $N(v)=(1+|v|) \ln (1+|v|)-|v|$ with Luxemburg norm has the Kottman constant $K\left(l^{(N)}\right)=N^{-1}(1) / N^{-1}(1 / 2)$, which answers M. M. Rao and Z. D. Ren's [8] problem.

Let $N(v)=(1+|v|) \ln (1+|v|)-|v|$, and let $l^{(N)}$ and $l^{N}$ be the corresponding Orlicz sequence spaces equipped with the Luxemburg norm and Orlicz norm, respectively. The following problem was raised in [8] (Problems 2.7 and 3.8): What are the packing constants of $l^{(N)}$ and $l^{N}$ ? We now present an answer. We first extend the theory of quantitative indices of Orlicz spaces.
\end{abstract}

\section{Index functions of $N$-functions. Let}

$$
\Phi(u)=\int_{0}^{|u|} \phi(t) d t \quad \text { and } \quad \Psi(v)=\int_{0}^{|v|} \psi(s) d s
$$

be a pair of complementary $N$-functions, Krasnosel'skiı̌ and Rutickiı̌ [5] studied the limit of the function $t \phi(t) / \Phi(t)$ as $t \rightarrow \infty$. Later, Rao and Ren [7] and Lindenstrauss and Tzafriri [6] systematically investigated the following related values which Ren [8], [10], [11] calls quantitative indices:

$$
\begin{array}{ll}
A_{\Phi}=\liminf _{t \rightarrow \infty} \frac{t \phi(t)}{\Phi(t)}, & B_{\Phi}=\limsup _{t \rightarrow \infty} \frac{t \phi(t)}{\Phi(t)}, \\
A_{\Phi}^{0}=\liminf _{t \rightarrow 0} \frac{t \phi(t)}{\Phi(t)}, & B_{\Phi}^{0}=\limsup _{t \rightarrow 0} \frac{t \phi(t)}{\Phi(t)}, \\
\bar{A}_{\Phi}=\inf _{t>0} \frac{t \phi(t)}{\Phi(t)}, & \bar{B}_{\Phi}=\sup _{t>0} \frac{t \phi(t)}{\Phi(t)} .
\end{array}
$$

2000 Mathematics Subject Classification: 46E30, 46A45.

Key words and phrases: Orlicz space, indices, packing constants, Kottman constants. 
The same six indices can be defined for $\Psi(v)$. The relationships among them are as follows (cf. [6], [7]):

Proposition 1.1. Let $\Phi, \Psi$ be a pair of complementary $N$-functions. Then

$$
\begin{aligned}
& \frac{1}{A_{\Phi}}+\frac{1}{B_{\Psi}}=1=\frac{1}{A_{\Psi}}+\frac{1}{B_{\Phi}}, \\
& \frac{1}{A_{\Phi}^{0}}+\frac{1}{B_{\Psi}^{0}}=1=\frac{1}{A_{\Psi}^{0}}+\frac{1}{B_{\Phi}^{0}}, \\
& \frac{1}{\bar{A}_{\Phi}}+\frac{1}{\bar{B}_{\Psi}}=1=\frac{1}{\bar{A}_{\Psi}}+\frac{1}{\bar{B}_{\Phi}} .
\end{aligned}
$$

In 1985, on the basis of Cleaver [1], Ren studied the upper and lower limits of the function $\Phi^{-1}(u) / \Phi^{-1}(2 u)$ as $u \rightarrow \infty$. Later, Rao and Ren [7] (p. 23) and Ren and Chen [11] defined the following "new" quantitative indices:

$$
\begin{array}{ll}
\alpha_{\Phi}=\liminf _{u \rightarrow \infty} \frac{\Phi^{-1}(u)}{\Phi^{-1}(2 u)}, & \beta_{\Phi}=\limsup _{u \rightarrow \infty} \frac{\Phi^{-1}(u)}{\Phi^{-1}(2 u)}, \\
\alpha_{\Phi}^{0}=\liminf _{u \rightarrow 0} \frac{\Phi^{-1}(u)}{\Phi^{-1}(2 u)}, & \beta_{\Phi}^{0}=\limsup _{u \rightarrow 0} \frac{\Phi^{-1}(u)}{\Phi^{-1}(2 u)}, \\
\bar{\alpha}_{\Phi}=\inf _{u>0} \frac{\Phi^{-1}(u)}{\Phi^{-1}(2 u)}, & \bar{\beta}_{\Phi}=\sup _{u>0} \frac{\Phi^{-1}(u)}{\Phi^{-1}(2 u)} .
\end{array}
$$

The six indices of $\Psi$ can be similarly defined. It is easy to check the following relations among the above indices:

TheOREM 1.2. Let $\Phi, \Psi$ be a pair of complementary $N$-functions. Then

$$
\begin{aligned}
& 2 \alpha_{\Phi} \beta_{\Psi}=1=2 \alpha_{\Psi} \beta_{\Phi}, \\
& 2 \alpha_{\Phi}^{0} \beta_{\Psi}^{0}=1=2 \alpha_{\Psi}^{0} \beta_{\Phi}^{0}, \\
& 2 \bar{\alpha}_{\Phi} \bar{\beta}_{\Psi}=1=2 \bar{\alpha}_{\Psi} \bar{\beta}_{\Phi} .
\end{aligned}
$$

For a further study of the geometric constants, we should directly investigate the functions which produce the quantitative indices.

TheORem 1.3. Let $\Phi$ be an $N$-function, $\phi(t)$ being its left derivative. Define

$$
F_{\Phi}(t)=\frac{t \phi(t)}{\Phi(t)}, \quad G_{\Phi}(c, u)=\frac{\Phi^{-1}(u)}{\Phi^{-1}(c u)} \quad(c>1) .
$$

Then $F_{\Phi}(t)$ is increasing (resp. decreasing) on $\left(0, \Phi^{-1}\left(u_{0}\right)\right]$ if and only if $G_{\Phi}(c, u)$ is increasing (resp. decreasing) on $\left(0, u_{0} / c\right]$ for every $c>1$.

To prove Theorem 1.3, we first introduce two auxiliary lemmas. 
LEMma 1.4. A continuous function $f$ is increasing if and only if both its left derivative $f_{-}^{\prime}$ and its right derivative $f_{+}^{\prime}$ are nonnegative.

LemMA 1.5. Let $\phi_{+}(t)$ be the right derivative of $\Phi$. Write $F_{\Phi_{+}}(t)=$ $t \phi_{+}(t) / \Phi(t)$. Then $F_{\Phi}(t)$ is increasing if and only if $F_{\Phi_{+}}(t)$ is increasing.

Proof of Theorem 1.3. Necessity. In view of Lemma 1.4, it suffices to show that both the left derivative $G_{\Phi}^{\prime}(c, t)$ and the right derivative $G_{\Phi_{+}}^{\prime}(c, t)$ of $G_{\Phi}(c, t)$ are nonnegative when $F_{\Phi}(t)$ is increasing.

In fact, for every $u \in\left(0, u_{0} / c\right]$, let $\Phi^{-1}(u)=t_{1}$ and $\Phi^{-1}(u)=t_{2}$. Then $0<t_{1}<t_{2} \leq \Phi^{-1}\left(u_{0}\right)$ and

$$
\begin{aligned}
G_{\Phi}^{\prime}(c, u) & =\frac{1}{\left[\Phi^{-1}(c u)\right]^{2}}\left\{\frac{\Phi^{-1}(c u)}{\phi\left[\Phi^{-1}(u)\right]}-\frac{c \Phi^{-1}(u)}{\phi\left[\Phi^{-1}(c u)\right]}\right\} \\
& =\frac{1}{t_{2}^{2} \phi\left(t_{1}\right) \phi\left(t_{2}\right)}\left[t_{2} \phi\left(t_{2}\right)-c t_{1} \phi\left(t_{1}\right)\right] \\
& =\frac{c u}{t_{2}^{2} \phi\left(t_{1}\right) \phi\left(t_{2}\right)}\left[\frac{t_{2} \phi\left(t_{2}\right)}{\Phi\left(t_{2}\right)}-\frac{t_{1} \phi\left(t_{1}\right)}{\Phi\left(t_{1}\right)}\right] \\
& =\frac{\Phi\left(t_{2}\right)}{t_{2}^{2} \phi\left(t_{1}\right) \phi\left(t_{2}\right)}\left[F_{\Phi}\left(t_{2}\right)-F_{\Phi}\left(t_{1}\right)\right] .
\end{aligned}
$$

Analogously we have

$$
G_{\Phi_{+}}^{\prime}(c, u)=\frac{\Phi\left(t_{2}\right)}{t_{2}^{2} \phi_{+}\left(t_{1}\right) \phi_{+}\left(t_{2}\right)}\left[F_{\Phi_{+}}\left(t_{2}\right)-F_{\Phi_{+}}\left(t_{1}\right)\right] .
$$

Since $F_{\Phi}\left(t_{2}\right)-F_{\Phi}\left(t_{1}\right) \geq 0$ and $F_{\Phi_{+}}\left(t_{2}\right)-F_{\Phi_{+}}\left(t_{1}\right) \geq 0$ for $0<t_{1}<t_{2} \leq$ $\Phi^{-1}\left(u_{0}\right)$ when $u \in\left(0, u_{0} / c\right]$ by Lemma 1.5 , one has $G_{\Phi}^{\prime}(c, u) \geq 0$ and $G_{\Phi_{+}}^{\prime}(c, u) \geq 0$.

Sufficiency. To show that $F_{\Phi}(t)$ is increasing, for arbitrary $0<t_{1}<$ $t_{2} \leq \Phi^{-1}\left(u_{0}\right)$, choose $c_{1}=\Phi\left(t_{2}\right) / \Phi\left(t_{1}\right)$ and $u_{1}=\Phi\left(t_{1}\right) \leq \Phi\left(u_{0}\right) / c_{1}$. Then $\left.G_{\Phi}^{\prime}\left(c_{1}, u\right)\right|_{u=u_{1}} \geq 0$. Thus, $F_{\Phi}\left(t_{2}\right)-F_{\Phi}\left(t_{1}\right) \geq 0$, which yields that $F_{\Phi}(t)$ is increasing on $\left(0, \Phi^{-1}\left(u_{0}\right)\right]$.

TheOREM 1.6. Let $\Phi, \Psi$ be a pair of complementary $N$-functions, let $\phi$ and $\psi$ be their left derivatives, respectively, and $C>0$. Let $_{F_{\Phi}}(t)$ and $F_{\Psi}(s)$ be defined as (13), i.e.,

$$
F_{\Phi}(t)=\frac{t \phi(t)}{\Phi(t)}, \quad F_{\Psi}(s)=\frac{s \psi(s)}{\Psi(s)}, \quad t, s>0 .
$$

Then

(i) $F_{\Phi}(t)$ is increasing (resp. decreasing) on $(0, \psi(C)]$ if and only if $F_{\Psi}(s)$ is decreasing (resp. increasing) on $(0, C]$.

(ii) Define

$$
a_{\Phi}^{*}=\inf \left\{F_{\Phi}(t): t \in(0, \psi(C)]\right\},
$$




$$
b_{\Psi}^{*}=\sup \left\{F_{\Psi}(s): s \in(0, C]\right\} .
$$

Then

$$
\frac{1}{a_{\Phi}^{*}}+\frac{1}{b_{\Psi}^{*}}=1
$$

Proof. (i) Since $\psi(s)$ is always increasing, the set of discontinuity points of $\psi(s)$ which are leap points is nondense and at most countable.

(A) Suppose $\psi(s)$ is continuous and strictly increasing on some subinterval $\left[s_{1}, s_{2}\right]$ of $(0, C]$. Then its inverse function $\phi(t)$ is continuous and strictly increasing on $\left[\psi\left(s_{1}\right), \psi\left(s_{2}\right)\right] \subset(0, \psi(C)]$, and hence $\phi(\psi(s))=s$. Therefore

$$
\begin{aligned}
F_{\Psi}(s) & =\frac{s \psi(s)}{\Psi(s)}=\frac{\phi(t) \psi(\phi(t))}{\Psi(\phi(t))}=\frac{\phi(t) t}{\Psi(\phi(t))} \\
& =\frac{t \phi(t)}{t \phi(t)-\Phi(t)}=\frac{t \phi(t) / \Phi(t)}{t \phi(t) / \Phi(t)-1}=\frac{F_{\Phi}(t)}{F_{\Phi}(t)-1},
\end{aligned}
$$

i.e.,

$$
\frac{1}{F_{\Psi}(s)}+\frac{1}{F_{\Phi}(\psi(s))}=1, \quad s \in\left[s_{1}, s_{2}\right] .
$$

(B) Suppose $\psi(s)$ is a constant $t_{0}$ on an interval $\left[s_{1}, s_{2}\right] \subset(0, C]$. Then for any $s_{1}^{\prime}, s_{2}^{\prime}$ satisfying $s_{1} \leq s_{1}^{\prime}<s_{2}^{\prime} \leq s_{2}$, we have

$$
\begin{aligned}
F_{\Psi}\left(s_{1}^{\prime}\right)-F_{\Psi}\left(s_{2}^{\prime}\right) & =\frac{s_{1}^{\prime} \psi\left(s_{1}^{\prime}\right)}{\Psi\left(s_{1}^{\prime}\right)}-\frac{s_{2}^{\prime} \psi\left(s_{2}^{\prime}\right)}{\Psi\left(s_{2}^{\prime}\right)} \\
& =\left[\frac{s_{1}^{\prime}}{\Psi\left(s_{1}^{\prime}\right)}-\frac{s_{2}^{\prime}}{\Psi\left(s_{2}^{\prime}\right)}\right] t_{0}>0 .
\end{aligned}
$$

This means $F_{\Psi}(s)$ is decreasing on $\left[s_{1}, s_{2}\right]$.

(C) Suppose $\psi(s)$ leaps at the point $s_{0}$. Let $\psi\left(s_{0}+0\right)-\psi\left(s_{0}-0\right)=k>0$. For any $\varepsilon>0$, there are $s_{1}^{\prime}, s_{2}^{\prime}$ satisfying $s_{1}^{\prime} \leq s_{0} \leq s_{2}^{\prime}$ and $0<s_{2}^{\prime}-s_{1}^{\prime}<\varepsilon$. We deduce that $\psi\left(s_{2}^{\prime}\right)-\psi\left(s_{1}^{\prime}\right) \geq k$, and

$$
\begin{aligned}
F_{\Psi}\left(s_{2}^{\prime}\right)-F_{\Psi}\left(s_{1}^{\prime}\right) & =\frac{s_{2}^{\prime} \psi\left(s_{2}^{\prime}\right)}{\Psi\left(s_{2}^{\prime}\right)}-\frac{s_{1}^{\prime} \psi\left(s_{1}^{\prime}\right)}{\Psi\left(s_{1}^{\prime}\right)} \\
& \geq\left[\frac{s_{2}^{\prime}}{\Psi\left(s_{2}^{\prime}\right)}-\frac{s_{1}^{\prime}}{\Psi\left(s_{1}^{\prime}\right)}\right] \psi\left(s_{1}^{\prime}\right)+\frac{s_{2}^{\prime}}{\Psi\left(s_{2}^{\prime}\right)} k .
\end{aligned}
$$

When $s_{2}^{\prime}-s_{1}^{\prime} \rightarrow 0$, we can see

$$
\frac{s_{2}^{\prime}}{\Psi\left(s_{2}^{\prime}\right)}-\frac{s_{1}^{\prime}}{\Psi\left(s_{1}^{\prime}\right)} \rightarrow 0, \quad \frac{s_{2}^{\prime}}{\Psi\left(s_{2}^{\prime}\right)} k \rightarrow \frac{s_{0}}{\Psi\left(s_{0}\right)} k>0,
$$

since the function $s / \Psi(s)$ is continuous. Thus, $F_{\Psi}\left(s_{2}^{\prime}\right)-F_{\Psi}\left(s_{1}^{\prime}\right)>0$, which implies that $F_{\Psi}(s)$ also leaps at the point $s_{0}$.

(D) Assume that $\psi(s)$ is a constant $t_{0}$ on some maximum interval $\left[s_{1}, s_{2}\right]$ (i.e. $\inf \left\{s: \psi(s)=t_{0}\right\}=s_{1}, \sup \left\{s: \psi(s)=t_{0}\right\}=s_{2}$ ). We know that $F_{\Psi}(s)$ 
decreases from $F_{\Psi}\left(s_{1}-0\right)$ to $F_{\Psi}\left(s_{2}+0\right)$ by (B). At the same time, $\phi(t)$ leaps at the point $t_{0}=\psi\left(s_{1}\right)=\psi\left(s_{2}\right)$. By $(\mathrm{C})$, we know $F_{\Phi}(t)$ also leaps from $F_{\Phi}\left(t_{0}-0\right)$ to $F_{\Phi}\left(t_{0}+0\right)$. Letting $s \rightarrow s_{1}-0$ resp. $s \rightarrow s_{2}+0$ in (17), we have

$$
\begin{aligned}
& \frac{1}{F_{\Psi}\left(s_{1}-0\right)}+\frac{1}{F_{\Phi}\left(t_{0}-0\right)}=1, \\
& \frac{1}{F_{\Psi}\left(s_{2}+0\right)}+\frac{1}{F_{\Phi}\left(t_{0}+0\right)}=1 .
\end{aligned}
$$

This makes it clear that when $F_{\Psi}(s)$ continuously decreases from $F_{\Psi}\left(s_{1}-0\right)$ to $F_{\Psi}\left(s_{2}+0\right), F_{\Phi}(t)$ leaps up from $F_{\Phi}\left(t_{0}-0\right)$ to $F_{\Phi}\left(t_{0}+0\right)$.

(E) When $\psi(s)$ leaps at the point $s_{0}, \phi(t)$ is a constant on the maximum interval $\left[\psi\left(s_{0}\right), \psi\left(s_{0}+0\right)\right]$. Therefore $F_{\Phi}(t)$ decreases on that interval by (B). As in (D), let $s \rightarrow s_{0}-0$ resp. $s \rightarrow s_{0}+0$ in (17) to find that

$$
\begin{aligned}
& \frac{1}{F_{\Psi}\left(s_{0}-0\right)}+\frac{1}{F_{\Phi}\left(\psi\left(s_{0}-0\right)\right)}=1, \\
& \frac{1}{F_{\Psi}\left(s_{0}+0\right)}+\frac{1}{F_{\Phi}\left(\psi\left(s_{0}+0\right)\right)}=1,
\end{aligned}
$$

which implies that when $F_{\Psi}(s)$ leaps up from $F_{\Psi}\left(s_{0}-0\right)$ to $F_{\Psi}\left(s_{0}+0\right), F_{\Phi}(t)$ continuously decreases from $F_{\Phi}\left(\psi\left(s_{0}-0\right)\right)$ to $F_{\Phi}\left(\psi\left(s_{0}+0\right)\right)$.

Consequently, we find from the above cases that $F_{\Psi}(s)$ increases (resp. decreases) if and only if $F_{\Phi}(t)$ decreases (resp. increases) on the corresponding intervals. The proof of (i) is complete.

(ii) From the discussion of (i) we know that if $\left[s_{1}, s_{2}\right]$ is a maximum interval of constancy of $\psi(s)$ in $(0, C]$ or an interval where $\psi(s)$ is strictly increasing continuous, we always have

$$
\begin{aligned}
\frac{1}{\sup \left\{F_{\Psi}(s): s \in\left[s_{1}-0, s_{2}+0\right]\right\}} & \\
& +\frac{1}{\inf \left\{F_{\Phi}(t): t \in\left[t_{1}-0, t_{2}+0\right]\right\}}=1,
\end{aligned}
$$

where $t_{1}-0=\psi\left(s_{1}-0\right), t_{2}+0=\psi\left(s_{2}+0\right)$. When $s_{0}$ is a leap point of $\psi(s)$, we treat it as an interval $\left[s_{0}, s_{0}\right]$, so that $(22)$ still holds. Combining the above intervals, we have

$$
\frac{1}{\sup \left\{F_{\Psi}(s): s \in(0, C]\right\}}+\frac{1}{\inf \left\{F_{\Phi}(t): t \in(0, \psi(C)]\right\}}=1,
$$

i.e., (16) holds as soon as $C$ is a strictly increasing continuous point or the right end of a maximum constancy interval of $\psi(s)$. It suffices to show that (16) holds when $C$ is an internal point of a maximum constancy interval of $\psi(s)$. In that case, let $s_{0}$ be the left end of that interval. Therefore, $F_{\Psi}(s)$ is decreasing on $\left[s_{0}, C\right]$, and hence

$$
b_{\Psi}^{*}=\sup \left\{F_{\Psi}(s): s \in(0, C]\right\}=\sup \left\{F_{\Psi}(s): s \in\left(0, s_{0}+0\right]\right\} .
$$


Meanwhile, $F_{\Phi}(t)$ leaps up at the point $\psi(C)$, so that

$$
a_{\Phi}^{*}=\inf \left\{F_{\Phi}(t): t \in(0, \psi(C)]\right\}=\inf \left\{F_{\Phi}(t): t \in\left(0, \psi\left(s_{0}+0\right)\right]\right\} .
$$

It follows that

$$
\frac{1}{\sup _{s \in\left(0, s_{0}+0\right]} F_{\Psi}(s)}+\frac{1}{\inf _{t \in\left(0, \psi\left(s_{0}+0\right)\right]} F_{\Phi}(t)}=1
$$

since (22) holds. Consequently, (16) holds in any case by (24)-(26).

\section{Packing in Orlicz sequence spaces with Luxemburg norm}

Definition $2.1([4],[8])$. The packing constant $P(X)$ of a Banach space $X$ is

$P(X)=\sup \{r>0$ : infinitely many balls of radius $r$

$$
\text { can be packed into the unit ball of } X\} \text {. }
$$

The Kottman constant of an infinite-dimensional Banach space $X$ is defined as

$$
K(X)=\sup \left\{\inf _{i \neq j}\left\|x_{i}-x_{j}\right\|:\left\{x_{i}\right\}_{i=1}^{\infty} \subset S(X)\right\}
$$

where $S(X)$ is the unit sphere of $X$.

Clearly, $1 \leq K(X) \leq 2$. The following relationship was proved by Kottman [4] (cf. Ye [14]):

Proposition 2.2. For an infinite-dimensional Banach space $X$, one has

$$
P(X)=\frac{K(X)}{2+K(X)} .
$$

Hudzik [3] verified that $K(X)=2$ if $X$ is a nonreflexive Banach lattice, therefore $P(X)=1 / 2$. Since $l^{(\Phi)}$ and $l^{\Phi}$ are Banach lattices, it suffices to consider the Kottman constant when $\Phi \in \Delta_{2}(0) \cap \nabla_{2}(0)$, i.e., when $l^{(\Phi)}$ and $l^{\Phi}$ are reflexive (we calculate the packing constants $P\left(l^{(\Phi)}\right)$ and $P\left(l^{\Phi}\right)$ from $(27))$.

In this section, we investigate $K\left(l^{(\Phi)}\right)$ of an Orlicz sequence space with Luxemburg norm and answer Rao and Ren's [8] Problem 2.7.

Lemma 2.3. For an $N$-function $\Phi, \Phi \in \Delta_{2}(0) \cap \nabla_{2}(0)$ implies

$$
\max \left(\frac{1}{\alpha_{\Phi}^{0}}, \frac{1}{\alpha_{\Phi}^{\prime}}\right) \leq K\left(l^{(\Phi)}\right) \leq \frac{1}{\widetilde{\alpha}_{\Phi}},
$$

where

$$
\begin{aligned}
& \alpha_{\Phi}^{\prime}=\inf \left\{\frac{\Phi^{-1}\left(\frac{1}{2 k}\right)}{\Phi^{-1}\left(\frac{1}{k}\right)}: k=1,2, \cdots\right\} \\
& \widetilde{\alpha}_{\Phi}=\inf \left\{\frac{\Phi^{-1}(u)}{\Phi^{-1}(2 u)}: 0<u \leq \frac{1}{2}\right\} .
\end{aligned}
$$


Proof. The inequality

$$
\frac{1}{\alpha_{\Phi}^{\prime}} \leq K\left(l^{(\Phi)}\right) \leq \frac{1}{\widetilde{\alpha}_{\Phi}}
$$

is due to Ye [14]. Rao and Ren [8, Theorem 2.3] proved

$$
1 / \alpha_{\Phi}^{0} \leq K\left(l^{(\Phi)}\right)
$$

so (28) holds in its entirety.

REMARK 2.4. Let

$$
\widetilde{A}_{\Phi}=\inf \left\{t \phi(t) / \Phi(t): 0<t \leq \Phi^{-1}(1)\right\} .
$$

By using the right side of (32), it is proved in [8] (Theorem 2.3) that

$$
2^{1 / B_{\Phi}^{0}} \leq K\left(l^{(\Phi)}\right) \leq 2^{1 / \widetilde{A}_{\Phi}} .
$$

If $F_{\Phi}(t)=t \phi(t) / \Phi(t)$ is increasing on $t \in(0, \infty)$, then $\widetilde{A}_{\Phi}=B_{\Phi}^{0}=C_{\Phi}^{0}=$ $\lim _{t \rightarrow 0} t \phi(t) / \Phi(t)$ and by (31) we have

$$
K\left(l^{(\Phi)}\right)=2^{1 / C_{\Phi}^{0}}
$$

For a pair of complementary $N$-functions

$$
M(u)=e^{|u|}-|u|-1 \quad \text { and } \quad N(v)=(1+|v|) \ln (1+|v|)-|v|,
$$

since $F_{M}(t)=t M^{\prime}(t) / M(t)$ is increasing on $(0, \infty)$, the authors of [8] computed that $K\left(l^{(M)}\right)=\sqrt{2}$ by $(32)$. However, $F_{N}(t)$ is decreasing on $(0, \infty)$. They only estimated (cf. (24) of [8]):

$$
1.41 \approx \sqrt{2} \leq K\left(l^{(N)}\right) \leq K\left(l^{N}\right) \leq 2^{1 /(e-1)} \approx 1.50 .
$$

They asked (see [8, Problem 2.7]): What is the exact value of $K\left(l^{(N)}\right)$ ? For the solution of the problem, we give the following lemma which follows from Theorem 1.3 by taking $c=2$.

Lemma 2.5. Let $\Phi$ be an $N$-function, $\phi(t)$ being its left derivative. Set

$$
F_{\Phi}(t)=\frac{t \phi(t)}{\Phi(t)}, \quad G_{\Phi}(u)=\frac{\Phi^{-1}(u)}{\Phi^{-1}(2 u)}
$$

for $t, u>0$ (see (13)). If $F_{\Phi}(t)$ is increasing (resp. decreasing) on $\left(0, \Phi^{-1}\left(u_{0}\right)\right]$, then $G_{\Phi}(u)$ is increasing (resp. decreasing) on $\left(0, u_{0} / 2\right]$.

Now we establish the following

Theorem 2.6. For $\Phi \in \Delta_{2}(0) \cap \nabla_{2}(0)$, we have:

(i) If $F_{\Phi}(t)=t \phi(t) / \Phi(t)$ is increasing on $\left(0, \Phi^{-1}(1)\right]$, then the Kottman constant of the Orlicz sequence space $l^{(\Phi)}$ equipped with the Luxemburg norm is

$$
K\left(l^{(\Phi)}\right)=2^{1 / C_{\Phi}^{0}}
$$

where $C_{\Phi}^{0}=\lim _{t \rightarrow 0} F_{\Phi}(t)$. 
(ii) If $F_{\Phi}(t)$ is decreasing on $\left(0, \Phi^{-1}(1)\right]$, then

$$
K\left(l^{(\Phi)}\right)=\frac{\Phi^{-1}(1)}{\Phi^{-1}(1 / 2)} .
$$

Proof. The proof of (i) has been given in Remark 2.4.

(ii) If $F_{\Phi}(t)$ is decreasing on $\left(0, \Phi^{-1}(1)\right]$, then $G_{\Phi}(u)$ is also decreasing on $(0,1 / 2]$ by Lemma 2.5. Therefore

$$
\alpha_{\Phi}^{\prime}=\widetilde{\alpha}_{\Phi}=\left.\left[G_{\Phi}(u)\right]\right|_{u=1 / 2}=\frac{\Phi^{-1}(1 / 2)}{\Phi^{-1}(1)} .
$$

Note that $\alpha_{\Phi}^{0} \geq \alpha_{\Phi}^{\prime}$, and (39) follows from Lemma 2.3 and the above.

ExAmple 2.7. Let $N(v)=(1+|v|) \ln (1+|v|)-|v|$. Then we have the exact value:

$$
K\left(l^{(N)}\right)=\frac{N^{-1}(1)}{N^{-1}(1 / 2)} \approx 1.48699, \quad P\left(l^{(N)}\right) \approx 0.42644 .
$$

Indeed, it is easy to see that

$$
F_{N}(t)=\frac{t N^{\prime}(t)}{N(t)}=\frac{t \ln (1+t)}{(1+t) \ln (1+t)-t}
$$

is decreasing on $(0, \infty)$ and $C_{N}^{0}=\lim _{t \rightarrow 0} F_{N}(t)=2$, i.e., $N \in \Delta_{2}(0) \cap \nabla_{2}(0)$. Hence, (41) follows from Theorem 2.6(ii). Formula (41) solves Problem 2.7 of $[8]$.

REMARK 2.8. The index function $F_{\Phi}(t)$ of an $N$-function is generally easy to find, and is monotonic. So the exact value of the packing constant of most Orlicz sequence spaces with Luxemburg norm can be computed in this way. For instance, for the $N$-function of Krasnosel'skil and Rutickiı [5, p. 30]

$$
\Phi(u)=\frac{u^{2}}{\ln (e+|u|)}
$$

we have

$$
K\left(l^{(\Phi)}\right)=\frac{\Phi^{-1}(1)}{\Phi^{-1}(1 / 2)} \approx 1.46987 \quad \text { and } \quad P\left(l^{(\Phi)}\right) \approx 0.42361
$$

Indeed, since the index function

$$
F_{\Phi}(t)=\frac{t \phi(t)}{\Phi(t)}=2-\frac{t}{(e+t) \ln (e+t)}
$$

is decreasing on $\left(0, \Phi^{-1}(1)\right]$ and $C_{\Phi}^{0}=2$, we have $\Phi \in \Delta_{2}(0) \cap \nabla_{2}(0)$. Thus (42) follows from Theorem 2.6(ii).

Another example is the $N$-function of Gallardo [2]:

$$
\Phi_{p, r}(u)=|u|^{p} \ln ^{r}(1+|u|), \quad 1 \leq p<\infty, 0<r<\infty .
$$


Then one has

$$
K\left(l^{\left(\Phi_{p, r}\right)}\right)=\frac{\Phi_{p, r}^{-1}(1)}{\Phi_{p, r}^{-1}(1 / 2)}
$$

In fact,

$$
F_{\Phi_{p, r}}(t)=p+\frac{r t}{(1+t) \ln (1+t)}
$$

for $t>0$. Since $C_{\Phi_{p, r}}^{0}=\lim _{t \rightarrow 0^{+}} F_{\Phi_{p, r}}(t)=p+r>1$ we deduce that $\Phi_{p, r} \in \Delta_{2}(0) \cap \nabla_{2}(0)$. On the other hand,

$$
\frac{d}{d t} F_{\Phi_{p, r}}(t)=\frac{r[\ln (1+t)-t]}{(1+t)^{2} \ln ^{2}(1+t)}<0
$$

for $t>0$, i.e., $F_{\Phi_{p, r}}(t)$ is decreasing on $\left(0, \Phi_{p, r}^{-1}(1)\right]$. Thus the exact value (43) of $K\left(l^{\left(\Phi_{p, r}\right)}\right)$ results from Theorem 2.6(ii). In particular, if $p=r=1$, i.e., $\Phi_{1,1}(u)=|u| \ln (1+|u|)$, then

$$
K\left(l^{\left(\Phi_{1,1}\right)}\right)=\frac{\Phi_{1,1}^{-1}(1)}{\Phi_{1,1}^{-1}(1 / 2)} \approx 1.18806 \quad \text { and } \quad P\left(l^{\left(\Phi_{1,1}\right)}\right) \approx 0.37266
$$

We now present an interpolation theorem which supplements the corresponding results in [8].

TheOREM 2.9. Let $\Phi$ be an $N$-function, $\Phi_{0}(u)=u^{2}, 0<s \leq 1$, and let $\Phi_{s}$ be the inverse of

$$
\Phi_{s}^{-1}(u)=\left[\Phi^{-1}(u)\right]^{1-s}\left[\Phi_{0}^{-1}(u)\right]^{s}, \quad 0<s \leq 1, u \geq 0 .
$$

Then

$$
\max \left(\frac{1}{\alpha_{\Phi_{s}}^{0}}, \frac{1}{\alpha_{\Phi_{s}}^{\prime}}\right) \leq K\left(l^{\left(\Phi_{s}\right)}\right) \leq \frac{1}{\widetilde{\alpha}_{\Phi_{s}}} \leq 2^{1-s / 2} .
$$

In particular, if $\Phi \notin \nabla_{2}(0)$, then

$$
K\left(l^{\left(\Phi_{s}\right)}\right)=2^{1-s / 2} .
$$

Therefore, the following assertions hold:

(i) If $F_{\Phi}(t)=t \phi(t) / \Phi(t)$ is increasing on $\left(0, \Phi^{-1}(1)\right]$, then

$$
K\left(l^{\left(\Phi_{s}\right)}\right)=2^{(1-s) / C_{\Phi}^{0}+s / 2} .
$$

(ii) If $F_{\Phi}(t)$ is decreasing on $\left(0, \Phi^{-1}(1)\right]$, then

$$
K\left(l^{\left(\Phi_{s}\right)}\right)=2^{s / 2}\left[\frac{\Phi^{-1}(1)}{\Phi^{-1}(1 / 2)}\right]^{1-s} .
$$

Proof. Ren [10] proved that $\Phi_{s} \in \Delta_{2}(0) \cap \nabla_{2}(0)$ for $0<s \leq 1$. To prove (46), by Lemma 2.3 we only need to show the rightmost inequality. Indeed, 
$\Phi^{-1}(u) / \Phi^{-1}(2 u) \geq 1 / 2$ for $u>0$ and hence,

$$
G_{\Phi_{s}}(u)=\left[\frac{\Phi^{-1}(u)}{\Phi^{-1}(2 u)}\right]^{1-s}\left[\frac{\sqrt{u}}{\sqrt{2 u}}\right]^{s}=2^{-s / 2}\left[G_{\Phi}(u)\right]^{1-s}, \quad u>0,
$$

which implies that

$$
\widetilde{\alpha}_{\Phi_{s}} \geq 2^{-s / 2}\left(\frac{1}{2}\right)^{1-s}=2^{s / 2-1} .
$$

If $\Phi \in \nabla_{2}(0)$, then $\alpha_{\Phi}^{0}=1 / 2$, so that

$$
\alpha_{\Phi_{s}}^{0}=\liminf _{u \rightarrow 0} G_{\Phi_{s}}(u)=\left(\alpha_{\Phi}^{0}\right)^{1-s} 2^{-s / 2}=2^{s / 2-1} .
$$

Thus, (47) follows from (52) and (46).

The hypothesis of (i) implies that $G_{\Phi}(u)$ is increasing on $(0,1 / 2]$ (cf. Lemma 2.5). It follows from (46) that

$$
\widetilde{\alpha}_{\Phi_{s}}=\alpha_{\Phi_{s}}^{0}=2^{-s / 2}\left[\lim _{u \rightarrow 0} G_{\Phi}(u)\right]^{1-s}=2^{-s / 2-(1-s) / C_{\Phi}^{0}} .
$$

Hence, (48) follows from (46) and (53).

Under the condition of (ii), $G_{\Phi}(u)$ is decreasing on $(0,1 / 2]$, so that

$$
\widetilde{\alpha}_{\Phi_{s}}=\alpha_{\Phi_{s}}^{\prime}=2^{-s / 2}\left[G_{\Phi}(u)\right]_{u=1 / 2}=2^{-s / 2}\left[\frac{\Phi^{-1}(1 / 2)}{\Phi^{-1}(1)}\right]^{1-s} .
$$

Finally, (49) holds by (46) and (54).

REMARK 2.10. By using an interpolation inequality, Rao and Ren $[8$, Theorem 4.3, 4.8] proved that

$$
K\left(l^{\left(\Phi_{s}\right)}\right) \leq 2^{1-s / 2} .
$$

From the proof of Theorem 2.9 we see that (55) can be derived from (52) without the use of the interpolation inequality.

Example 2.11. Let $\Phi_{r}(u)=e^{|u|^{r}}-1$ with $1<r<\infty$. The inverse function of $\Phi_{s}$ is

$\Phi_{s}^{-1}(u)=\left[\Phi_{r}^{-1}(u)\right]^{1-s}\left[\Phi_{0}^{-1}(u)\right]^{s}=u^{s / 2}[\ln (1+u)]^{(1-s) / r}, \quad u>0,0<s \leq 1$.

Then the Kottman constant of $l^{\left(\Phi_{s}\right)}$ is

$$
K\left(l^{\left(\Phi_{s}\right)}\right)=2^{(1-s) / r+s / 2} .
$$

Indeed, $\Phi_{r} \in \nabla_{2}(0)$ since

$$
C_{\Phi_{r}}^{0}=\lim _{t \rightarrow 0} \frac{r t^{r} e^{t^{r}}}{e^{t^{r}}-1}=r .
$$

Note that

$$
G_{\Phi_{r}}(u)=\left[\frac{\ln (1+u)}{\ln (1+2 u)}\right]^{1 / r}
$$


is increasing on $(0, \infty) \supset(0,1 / 2] \cup\left(0,\left(Q_{\Phi_{s}}-1\right) / 2\right]$. From (51) and (46) we have

$$
\widetilde{\alpha}_{\Phi_{s}}=\alpha_{\Phi_{s}}^{0}=2^{-s / 2}\left(\alpha_{\Phi_{r}}^{0}\right)^{1-s}=2^{-s / 2-(1-s) / r}
$$

and

$$
K\left(l^{\left(\Phi_{s}\right)}\right)=2^{(1-s) / r+s / 2} .
$$

REMARK 2.12. Example 2.11 (56) strengthens the estimate

$$
2^{(1-s) / r+s / 2} \leq K\left(l^{\left(\Phi_{s}\right)}\right) \leq 2^{1-s / 2}
$$

of $[8$, Example 4.4] to an equality.

3. Packing in Orlicz sequence spaces with Orlicz norm. Let $N(v)=(1+|v|) \ln (1+|v|)-|v|$, and let $l^{N}$ be the Orlicz sequence space generated by $N(v)$ equipped with the Orlicz norm. Rao and Ren [8, Example 2.7 and Problem 3.8] estimated

$$
1.41 \leq K\left(l^{N}\right) \leq 1.56
$$

and asked what is the exact value of $K\left(l^{N}\right)$. In this section, we present some supplementary results to [8] and improve the estimate (57).

Lemma 3.1. Let $\Phi, \Psi$ be a pair of complementary $N$-functions, with $\Phi \in \Delta_{2}(0) \cap \nabla_{2}(0)$. Then the Kottman constant $K\left(l^{\Phi}\right)$ of the Orlicz sequence space $l^{\Phi}$ equipped with the Orlicz norm can be estimated as follows:

$$
\max \left(2 \beta_{\Psi}^{0}, 2 \beta_{\Psi}^{\prime}\right) \leq K\left(l^{\Phi}\right) \leq \frac{1}{\alpha_{\Phi}^{*}},
$$

where

$$
\begin{aligned}
& \beta_{\Psi}^{\prime}=\sup \left\{\frac{\Psi^{-1}(1 / 2 k)}{\Psi^{-1}(1 / k)}: k=1,2, \ldots\right\} \\
& \alpha_{\Phi}^{*}=\inf \left\{\frac{\Phi^{-1}(u)}{\Phi^{-1}(2 u)}: 0<u \leq \frac{1}{2}\left(Q_{\Phi}-1\right)\right\}
\end{aligned}
$$

with

$$
Q_{\Phi}=\sup _{\|x\|_{\Phi}=1}\left\{k_{x}>1:\|x\|_{\Phi}=\frac{1}{k_{x}}\left[1+\rho_{\Phi}\left(k_{x} x\right)\right]\right\} .
$$

Proof. Using Wang's [12] result, Rao and Ren [8] proved that $K\left(l^{\Phi}\right) \leq$ $1 / \alpha_{\Phi}^{*}$ for $\Phi \in \Delta_{2}(0)$ and $2 \beta_{\Psi}^{0} \leq K\left(l^{\Phi}\right)$ for any $N$-function $\Phi$. It follows that if $\Phi \notin \nabla_{2}(0)$ then $\beta_{\Psi}^{0}=1, K\left(l^{\Phi}\right)=2$. Thus, to show (58), it suffices to prove that

$$
2 \beta_{\Psi}^{\prime} \leq K\left(l^{\Phi}\right)
$$

For any given $k \geq 1$, let

$$
Z_{k}=(0, \ldots, 0) \quad \text { and } \quad X_{k}=\left[k \Psi^{-1}(1 / k)\right]^{-1}(1, \ldots, 1),
$$


with $\operatorname{dim} Z_{k}=\operatorname{dim} X_{k}=k$, and define

$$
x_{i}=\left(Z_{k}, \ldots, Z_{k}, X_{k}, Z_{k}, Z_{k}, \ldots\right), \quad i \geq 1,
$$

with $X_{k}$ being at the $i$ th position. Then $\left\|x_{i}\right\|_{\Phi}=1, i \geq 1$, and for $i \neq j$,

$$
\left\|x_{i}-x_{j}\right\|_{\Phi}=\frac{1}{k \Psi^{-1}(1 / k)}\left[2 k \Psi^{-1}\left(\frac{1}{2 k}\right)\right]=\frac{2 \Psi^{-1}(1 /(2 k))}{\Psi^{-1}(1 / k)},
$$

so that

$$
K\left(l^{\Phi}\right) \geq \inf _{i \neq j}\left\|x_{i}-x_{j}\right\|_{\Phi}=\frac{2 \Psi^{-1}(1 /(2 k))}{\Psi^{-1}(1 / k)} .
$$

Since $k \geq 1$ is arbitrary, by (59), one has

$$
K\left(l^{\Phi}\right) \geq \sup \left\{\frac{2 \Psi^{-1}(1 /(2 k))}{\Psi^{-1}(1 / k)}: k=1,2, \ldots\right\}=2 \beta_{\Psi}^{\prime} .
$$

Corollary $3.2([8])$. Let $\Phi \in \Delta_{2}(0) \cap \nabla_{2}(0)$, and $\Psi$ be its complementary $N$-function. Then

$$
1<2^{1 / B_{\Phi}^{0}} \leq K\left(l^{\Phi}\right) \leq 2^{1 / A_{\Phi}^{*}}<2,
$$

where

$$
A_{\Phi}^{*}=\inf \left\{\frac{t \phi(t)}{\Phi(t)}: 0<t \leq \Phi^{-1}\left(Q_{\Phi}-1\right)\right\} .
$$

In particular, if $F_{\Phi}(t)=t \phi(t) / \Phi(t)$ is increasing on $\left(0, \Phi^{-1}\left(Q_{\Phi}-1\right)\right]$, then $A_{\Phi}^{*}=B_{\Phi}^{0}=C_{\Phi}^{0}=\lim _{t \rightarrow 0} t \phi(t) / \Phi(t)$, and hence

$$
K\left(l^{\Phi}\right)=2^{1 / C_{\Phi}^{0}} .
$$

For the constant $Q_{\Phi}$ of the space $l^{\Phi}$ appearing in (60) and (64), one has $1 \leq Q_{\Phi} \leq \infty$. Wang [12] and $\mathrm{Wu}$ [13] proved: $\Phi \in \nabla_{2}(0)$ if and only if $Q_{\Phi}<\infty$. We give a better estimate of $Q_{\Phi}$.

Lemma 3.3. Let $\Phi, \Psi$ be a pair of complementary $N$-functions, with $\Phi \in \nabla_{2}(0)$. Then

$$
Q_{\Phi} \leq \frac{a_{\Phi}^{*}}{a_{\Phi}^{*}-1}
$$

where

$$
a_{\Phi}^{*}=\inf \left\{\frac{t \phi(t)}{\Phi(t)}: 0<t \leq \psi\left[\Psi^{-1}(1)\right]\right\} .
$$

Proof. Note that $\Phi \in \nabla_{2}(0)$ if and only if $A_{\Phi}^{0}=\liminf _{t \rightarrow 0} t \phi(t) / \Phi(t)$ $>1$. For every $0<t \leq \psi\left[\Psi^{-1}(1)\right]$ one has $t \phi(t) / \Phi(t)>1$, so that $a_{\Phi}^{*}>1$ for $\Phi \in \nabla_{2}(0)$. Observe that $a_{\Phi}^{*} \leq t_{0} \phi\left(t_{0}\right) / \Phi\left(t_{0}\right)<\infty$, where $t_{0}=\psi\left[\Psi^{-1}(1)\right]$. Now we show (66), which implies $Q_{\Phi}<\infty$ for $\Phi \in \nabla_{2}(0)$. 
For any given $x=(x(i)) \in l^{\Phi}$ with $\|x\|_{\Phi}=1$, since

$$
k_{x}^{* *}=\sup \left\{k_{x}>1: \sum_{i=1}^{\infty} \Psi\left[\phi\left(k_{x}|x(i)|\right)\right] \leq 1\right\},
$$

one has

$$
\sum_{i=1}^{\infty} \Psi\left[\phi\left(\left(k_{x}^{* *}-\varepsilon\right)|x(i)|\right)\right] \leq 1
$$

for all $\varepsilon>0$, so that $\left(k_{x}^{* *}-\varepsilon\right)|x(i)| \leq \psi\left[\Psi^{-1}(1)\right]$ for any $i \geq 1$. By definition of $a_{\Phi}^{*}$,

$$
a_{\Phi}^{*} \Phi\left[\left(k_{x}^{* *}-\varepsilon\right)|x(i)|\right] \leq\left(k_{x}^{* *}-\varepsilon\right)|x(i)| \phi\left[\left(k_{x}^{* *}-\varepsilon\right)|x(i)|\right], \quad i \geq 1 .
$$

It follows that

$$
\begin{aligned}
1 & \geq \sum_{i=1}^{\infty} \Psi\left\{\phi\left[\left(k_{x}^{* *}-\varepsilon\right)|x(i)|\right]\right\} \\
& =\sum_{i=1}^{\infty}\left\{\left(k_{x}^{* *}-\varepsilon\right)|x(i)| \phi\left[\left(k_{x}^{* *}-\varepsilon\right)|x(i)|\right]-\Phi\left[\left(k_{x}^{* *}-\varepsilon\right)|x(i)|\right]\right\} \\
& \geq\left(a_{\Phi}^{*}-1\right) \sum_{i=1}^{\infty} \Phi\left[\left(k_{x}^{* *}-\varepsilon\right)|x(i)|\right] \\
& \geq\left(a_{\Phi}^{*}-1\right)\left[\left\|\left(k_{x}^{* *}-\varepsilon\right) x\right\|_{\Phi}-1\right] \\
& =\left(a_{\Phi}^{*}-1\right)\left[\left(k_{x}^{* *}-\varepsilon\right)-1\right],
\end{aligned}
$$

i.e.,

$$
k_{x}^{* *} \leq \frac{a_{\Phi}^{*}}{a_{\Phi}^{*}-1}+\varepsilon,
$$

which proves (66) since $Q_{\Phi}=\sup _{\|x\|_{\Phi}=1} k_{x}^{* *}$ and $\varepsilon$ is arbitrary.

REMARK 3.4. The constant $a_{\Phi}^{*}$ was introduced by Rao and Ren (cf. (36) of [8]). The estimate (66) is an improvement of [8, Remark 3.4]. Wu, Chen and Wang [13, Lemma 2(b)] gave the following estimate of $Q_{\Phi}$ :

$$
Q_{\Phi} \leq \max \left(2, \frac{2}{a_{\Phi}^{*}-1}\right)= \begin{cases}2, & a_{\Phi}^{*} \geq 2, \\ 2 /\left(a_{\Phi}^{*}-1\right), & 1<a_{\Phi}^{*}<2 .\end{cases}
$$

(66) also improves the above estimate. In fact, since $a_{\Phi}^{*} /\left(a_{\Phi}^{*}-1\right)<2$ if and only if $a_{\Phi}^{*}>2$, and $a_{\Phi}^{*} /\left(a_{\Phi}^{*}-1\right)<2 /\left(a_{\Phi}^{*}-1\right)$ for $1<a_{\Phi}^{*}<2$, we see that $a_{\Phi}^{*} \neq 2$ implies

$$
\frac{a_{\Phi}^{*}}{a_{\Phi}^{*}-1}<\max \left(2, \frac{2}{a_{\Phi}^{*}-1}\right)
$$

Corollary 3.5. For any $N$-function $\Phi$, we have

$$
Q_{\Phi} \leq b_{\Psi}^{*},
$$


where

$$
b_{\Psi}^{*}=\sup \left\{\frac{s \psi(s)}{\Psi(s)}: 0<s \leq \Psi^{-1}(1)\right\} .
$$

Proof. If $\Phi \notin \nabla_{2}(0), \Psi \notin \Delta_{2}(0)$, and $b_{\Psi}^{*} \geq B_{\Psi}^{0}=\limsup _{s \rightarrow 0} s \psi(s) / \Psi(s)$ $=\infty$, then (68) naturally holds. If $\Phi \in \nabla_{2}(0), \Psi \in \Delta_{2}(0)$, and $b_{\Psi}^{*}<\infty$,

$$
\frac{1}{a_{\Phi}^{*}}+\frac{1}{b_{\Psi}^{*}}=1
$$

i.e., $a_{\Phi}^{*} /\left(a_{\Phi}^{*}-1\right)=b_{\Psi}^{*}$ by Theorem 1.5(ii). Therefore (68) follows from (66).

THEOREM 3.6. Let $\Phi, \Psi$ be a pair of complementary $N$-functions with $\Phi \in \Delta_{2}(0) \cap \nabla_{2}(0)$.

(i) If $F_{\Phi}(t)=t \phi(t) / \Phi(t)$ is increasing on $\left(0, \Phi^{-1}\left(b_{\Psi}^{*}-1\right)\right]$, then

$$
K\left(l^{\Phi}\right)=2^{1 / C_{\Phi}^{0}}, \quad C_{\Phi}^{0}=\lim _{t \rightarrow 0^{+}} F_{\Phi}(t) .
$$

(ii) If $F_{\Phi}(t)$ is decreasing on $\left(0, \psi\left[\Psi^{-1}(1)\right]\right]$, then

$$
\frac{2 \Psi^{-1}(1 / 2)}{\Psi^{-1}(1)} \leq K\left(l^{\Phi}\right) \leq \frac{\psi\left[\Psi^{-1}(1)\right]}{\Phi^{-1}\left\{\frac{1}{2} \Phi\left(\psi\left[\Psi^{-1}(1)\right]\right)\right\}} .
$$

Proof. (i) From Corollary 3.5, $F_{\Phi}(t)$ is increasing on $\left(0, \Phi^{-1}\left(Q_{\Phi}-1\right)\right]$, and (71) follows from Corollary 3.2 (61).

(ii) By Theorem 1.6(i), $F_{\Psi}(s)$ is increasing on $\left(0, \Psi^{-1}(1)\right]$. It follows from Theorem 1.3 that $G_{\Psi}(v)$ is increasing on $(0,1 / 2]$, and $G_{\Phi}(u)$ is decreasing on $\left(0, \frac{1}{2} \Phi\left(\psi\left[\Psi^{-1}(1)\right]\right)\right]$ and hence

$$
\begin{aligned}
b_{\Psi}^{*} & =\left[F_{\Psi}(s)\right]_{s=\Psi^{-1}(1)}=\Psi^{-1}(1) \psi\left[\Psi^{-1}(1)\right] \\
& =\Phi\left\{\psi\left[\Psi^{-1}(1)\right]\right\}+\Psi\left[\Psi^{-1}(1)\right]=\Phi\left\{\psi\left[\Psi^{-1}(1)\right]\right\}+1,
\end{aligned}
$$

i.e.,

$$
b_{\Psi}^{*}-1=\Phi\left\{\psi\left[\Psi^{-1}(1)\right]\right\} .
$$

It follows that

$$
\left(0, \frac{1}{2}\left(Q_{\Phi}-1\right)\right] \subset\left(0, \frac{1}{2}\left(b_{\Psi}^{*}-1\right)\right]=\left(0, \frac{1}{2} \Phi\left(\psi\left[\Psi^{-1}(1)\right]\right)\right] .
$$

Since $G_{\Phi}(u)$ is decreasing, one has

$$
\alpha_{\Phi}^{*}=\frac{\Phi^{-1}\left\{\frac{1}{2}\left(Q_{\Phi}-1\right)\right\}}{\Phi^{-1}\left(Q_{\Phi}-1\right)} \geq \frac{\Phi^{-1}\left\{\frac{1}{2} \Phi\left(\psi\left[\Psi^{-1}(1)\right]\right)\right\}}{\psi\left[\Psi^{-1}(1)\right]} .
$$

Then by Lemma 3.1,

$$
K\left(l^{\Phi}\right) \leq \frac{1}{\alpha_{\Phi}^{*}} \leq \frac{\psi\left[\Psi^{-1}(1)\right]}{\Phi^{-1}\left\{\frac{1}{2} \Phi\left(\psi\left[\Psi^{-1}(1)\right]\right)\right\}} .
$$


On the other hand, again by Theorem $1.3, G_{\Psi}(v)$ is increasing on $(0,1 / 2]$, thus

$$
K\left(l^{\Phi}\right) \geq \max \left(2 \beta_{\Psi}^{0}, 2 \beta_{\Psi}^{\prime}\right)=2 \beta_{\Psi}^{\prime}=\frac{2 \Psi^{-1}(1 / 2)}{\Psi^{-1}(1)} .
$$

Finally, (72) follows from (76) and (77).

Example 3.7 (Rao and Ren [8, Problem 3.8]). Let

$$
M(u)=e^{|u|}-|u|-1, \quad N(v)=(1+|v|) \ln (1+|v|)-|v| .
$$

Then $F_{N}(t)=t N^{\prime}(t) / N(t)$ is decreasing on $(0, \infty)$. Now we estimate $K\left(l^{N}\right)$ by Theorem 3.6.

Note that $M^{\prime}(u)=e^{u}-1$. Let $v_{0}=M^{-1}(1)$ and $v_{1}=M^{-1}(1 / 2)$. Then $v_{0}$ is a root of $e^{v_{0}}-v_{0}-1=1$, and $v_{1}$ is a root of $e^{v_{1}}-v_{1}-1=1 / 2$. We have $M^{\prime}\left[M^{-1}(1)\right]=e^{v_{0}}-1=v_{0}+1$, and hence by (72) one gets

$$
\frac{2 v_{1}}{v_{0}} \leq K\left(l^{N}\right) \leq \frac{v_{0}+1}{N^{-1}\left[\frac{1}{2} N\left(v_{0}+1\right)\right]} .
$$

By a simple computation, $v_{0}=1.14619, v_{1}=0.85767$, therefore,

$$
1.496 \leq K\left(l^{N}\right) \leq 1.498
$$

This improves the estimate of Rao and Ren [8, Example 3.7]: $1.41 \leq K\left(l^{N}\right)$ $\leq 1.56$.

The author would like to thank Prof. Z. D. Ren for his advice and help.

\section{References}

[1] C. E. Cleaver, Packing spheres in Orlicz spaces, Pacific J. Math. 65 (1976), 325-335.

[2] D. Gallardo, Orlicz spaces for which the Hardy-Littlewood maximal operator is bounded, Publ. Mat. 32 (1988), 261-266.

[3] H. Hudzik, Every nonreflexive Banach lattice has the packing constant equal to 1/2, Collect. Math. 44 (1993), 129-134.

[4] C. A. Kottman, Packing and reflexivity in Banach spaces, Trans. Amer. Math. Soc. 150 (1970), 565-576.

[5] M. A. Krasnosel'skiı̌ and Ya. B. Rutickiǔ, Convex Functions and Orlicz Spaces, Noordhoff, Groningen, 1961.

[6] J. Lindenstrauss and L. Tzafriri, Classical Banach Spaces, I and II, Springer, Berlin, 1977 and 1979

[7] M. M. Rao and Z. D. Ren, Theory of Orlicz Spaces, Dekker, New York, 1991.

[8] —, - Packing in Orlicz sequence spaces, Studia Math. 126 (1997), 235-251.

[9] Z. D. Ren, Packing in Orlicz function space with Luxemburg norm, J. Xiangtan Univ. 1 (1985), 51-60.

[10] -, Packing in Orlicz function spaces, Ph.D. Dissertation, Univ. of California, Riverside, 1994.

[11] Z. D. Ren and S. T. Chen, Jung constants of Orlicz function spaces, Collect. Math. 48 (1997), 743-771. 
[12] T. F. Wang, Packing constants of Orlicz sequence spaces, Chinese Ann. Math. Ser. A 8 (1987), 508-513 (in Chinese).

[13] C. X. Wu, S. T. Chen and Y. W. Wang, Geometric characters of Orlicz sequence spaces, J. Northeastern Math. 2 (1986), no. 1, 49-57.

[14] Y. N. Ye, Packing spheres in Orlicz spaces, Chinese Ann. Math. Ser. A 4 (1983), 487-493 (in Chinese).

Department of Mathematics

Suzhou University

Suzhou, Jiangsu, 215006, P.R. China

E-mail: yanyq@pub.sz.jsinfo.net

Received July 24, 2000

Revised version February 21, 2001 